\title{
Targeting self- and neoepitopes with a modular self-adjuvanting cancer vaccine
}

\author{
Elodie Belnoue, ${ }^{1}$ Jean-François Mayol,, Susanna Carboni, ${ }^{1}$ Wilma Di Berardino Besson, ${ }^{1}$ \\ Eloise Dupuychaffray, ${ }^{1}$ Annika Nelde, ${ }^{2}$ Stefan Stevanovic, ${ }^{2}$ Marie-Laure Santiago-Raber, ${ }^{1}$ \\ Paul R. Walker, ${ }^{3}$ and Madiha Derouazi ${ }^{1}$
}

'AMAL Therapeutics, Geneva, Switzerland. ${ }^{2}$ University of Tübingen, Interfaculty Institute for Cell Biology, Department of Immunology, Tubingen, Germany. ${ }^{3}$ Centre for Translational Research in Onco-Hematology, Department of Internal Medicine Specialties, University of Geneva and Division of Oncology, Geneva University Hospitals, Geneva, Switzerland.

\begin{abstract}
Induction of a potent CD4 and CD8 T cell response against tumor-specific and tumor-associated antigens is critical for eliminating tumor cells. Recent vaccination strategies have been hampered by an inefficacious and low-amplitude immune response. In this article, we describe a self-adjuvanted chimeric protein vaccine platform to address these challenges, characterized by a multidomain construction incorporating (a) a cell-penetrating peptide allowing internalization of several multiantigenic MHC-restricted peptides within (b) the multiantigenic domain and (c) a TLR2/4 agonist domain. Functionality of the resulting chimeric protein is based on the combined effect of the above-mentioned 3 different domains for simultaneous activation of antigen-presenting cells and antigen cross-presentation, leading to an efficacious multiantigenic and multiallelic cellular immune response. Helper and cytotoxic T cell responses were observed against model-, neo-, and self-antigens and were highly potent in several murine tumor models. The safety and the immunogenicity of a human vaccine candidate designed for colorectal cancer treatment was demonstrated in a nonhuman primate model. This therapeutic vaccine approach, which we believe to be newly engineered, is promising for the treatment of poorly infiltrated tumors that do not respond to currently marketed immunotherapies.
\end{abstract}

Authorship note: EB and JFM contributed equally to this work.

Conflict of interest: EB, JFM, WDBB, SC, ED, MLSR, and MD are employees of AMAL Therapeutics. MD has ownership interests in AMAL Therapeutics. MD and PRW are the inventors of patents related to the CPP (US 9,187,534 and PCT/ IB2013/058497). MD and EB are inventors in patents related to KISIMA (PCT/EP2016/000471). PRW is a consultant/advisory board member for AMAL Therapeutics.

Copyright: () 2019 American Society for Clinical Investigation

Submitted: January 9, 2019

Accepted: April 19, 2019

Published: June 6, 2019.

Reference information: JCI Insight. 2019;4(11):e127305. https://doi. org/10.1172/ji.insight.127305.

\section{Introduction}

Although the immune system can recognize and to a certain extent eliminate tumor cells, the antitumor immune response often remains inefficient and of low amplitude. Protein-based therapeutic vaccines aim to boost this immune response by inducing a robust and prolonged cellular immune response against specific tumor-associated antigens. However, although supported by extremely promising preclinical data, cancer vaccines have, so far, only achieved moderate clinical efficacy $(1,2)$. This discrepancy highlights the need to develop a new class of vaccines with improved properties that induce a more potent antitumoral response.

One of the four key issues preventing therapeutic cancer vaccines from eradicating cancer cells is caused by the heterogenic nature of individual tumors. These tumors are composed of multiple cancer cell clones expressing varying levels of different tumor-specific and tumor-associated antigens (TSA and TAA), leading to variable immunogenicity within the same lesion $(3,4)$. Tumor immunogenicity is also shaped by both naturally occurring and vaccine-induced immune pressure, through the emergence of antigen-loss variants that favor cancer relapse (5). Therefore, in order to achieve a strong and durable immune response, therapeutic cancer vaccines should comprise multiple carefully selected TSA and/or TAA antigens (and preferably multiple epitopes of each TSA/TAA) with multiallelic HLA restriction of epitopes enabling treatment of a broad range of patients.

A second issue is that many tumors have low levels of infiltrating immune cells, which limits antitumoral efficacy. Therapeutic vaccines are becoming a key element for increasing immune cell infiltration in poorly infiltrated tumors (6), which offer the additional benefit of potentiating antitumor efficacy of immune checkpoint-modulating agents (7).

A third desired property of cancer vaccines is the ability to induce a complete cellular immune response involving both tumor-specific CD8 cytotoxic and CD4 helper T cells (2). However, antigens derived from conventional soluble protein- and peptide-based vaccines are only poorly cross-presented on MHC class I molecules by DCs and therefore induce only low-level CD8 T cell responses $(8,9)$. 
We have previously demonstrated that this major drawback can be overcome by using cell-penetrating peptides (CPPs) as delivery vectors $(10,11)$. These small peptides can cross the plasma membrane even when linked to large multiepitopic cargos, allowing antigens to be delivered into different processing compartments of DCs, so that they are presented on both MHC class I and class II, promoting CD8 and CD4 T cell activation, respectively (10).

Adjuvanticity represents a fourth critical aspect of therapeutic cancer vaccine development. Crucially, activation of TLRs through binding of an appropriate ligand leads to the maturation of DCs, and consequently, to the initiation of an efficacious antigen-specific $\mathrm{T}$ cell response. Conjugating a vaccine to a TLR ligand was explored with various TLR ligands combined to a peptide or protein vaccine and resulted in improved antigen-specific CD8 T cell responses (12). Based on these findings, we optimized our CPP-based vaccine and recently demonstrated that part of the efficacy of CPP-based therapeutic cancer vaccines relies on their combination with the concomitant administration of the optimal adjuvant (11). A further enhancement of immune activation would be to conjugate the protein vaccine to an appropriate TLR ligand (13) as a state-of-the-art recombinant fusion protein vaccine construct, thereby benefitting from self-adjuvanticity.

We herein report the development of an original chimeric protein vaccine platform, named KISIMA, to address these challenges. KISIMA is composed of 3 elements: a ZEBRA-derived CPP, a multiantigenic domain (Mad) with epitopes restricted by multiple MHC alleles, and a self-adjuvanting TLR2/4 agonist. Using a range of preclinical mouse tumor models, we demonstrate that within our improved and potentially unique vaccine construct, the CPP and the TLR agonist (TLRag) act together to elicit both CD8 and CD4 antigen-specific immune responses. This leads to immunological memory and high vaccine efficacy with increased intratumoral leukocyte infiltration. Importantly, we show that these vaccines are safe and elicit a break of tolerance and antigen-specific $\mathrm{T}$ cell immune responses in nonhuman primates (NHPs), attesting to the safety and immune efficacy of the platform for future clinical use.

\section{Results}

The self-adjuvanted KISIMA cancer vaccine platform. Different vaccine constructs were generated incorporating TLRags along with the CPP and Mad domains (Figure 1A). CD8 and CD4 epitopes of the different multiantigenic domains that were generated with their sequences presented are summarized in Supplemental Table 1 (supplemental material available online with this article at https://doi.org/10.1172/ jci.insight.127305DS1). Various constructs were produced in order to define the optimal self-adjuvanting module and to determine whether the position of the module within the construct affects its activity (Figure 1A). For instance, 2 of the constructs comprised Z13 CPP, Mad5, and 1 of the 2 TLRags (either at the C-or N-terminus): (a) Anaxa, a TLR2-4 peptide agonist, which originates from the N-terminus of annexin II or (b) a TLR4 agonist called extra domain A (EDA) from fibronectin (14). Upon vaccination, EDA at the N-terminus position elicited the highest $\mathrm{T}$ cell immune response against ovalbumin, whereas Anaxa was more efficacious at the C-terminus position (data not shown). The constructs with the optimal position of TLRags were thus compared for adjuvanting activity in human embryonic kidney (HEK) cells expressing human TLR2 or TLR4 and in THP1-XBlue-MD2-CD14 cells. The Anaxa module showed a higher activation capacity compared to EDA for both TLR2, as expected, and TLR4 activation as observed in Figure 1B. Maximal activation required the presence of both elements, CPP and TLRags, namely, Z13 and Anaxa respectively, when compared with vaccines lacking either TLRags (Z13Mad5 construct) or CPP (Mad5Anaxa construct) demonstrating potentiation of the TLRag activity by the CPP for optimal effect (Figure 1, B and C) and confirming an activation pathway through both TLR molecules (Figure 1B). Moreover, a complete lack of activation of TLR4-knockout DCs by Z13Mad5Anaxa showed that the TLR4 pathway was necessary and sufficient for vaccine-mediated DC activation, whereas the TLR2 pathway was less critical (Supplemental Figure 1A). Surface plasmon resonance studies of ATP125, a vaccine containing human antigens (Figure 1A), confirmed affinity of vaccine for TLR4 and TLR2, but not for TLR3 (Figure 1D).

$A$ self-adjuvanted cancer vaccine platform eliciting CD8 and CD4 T cell immune responses. The constructs were then compared in vivo in an EG7 mouse thymoma model. When compared with EDAZ13Mad5, Z13Mad5Anaxa showed the strongest antitumor effect (Figure 2A); therefore, Anaxa was selected as the optimal TLRag for the next steps. Importantly, the antitumor activity of the vaccine was decreased when CPP Z13 was removed (Mad5Anaxa construct) (Figure 2B and Supplemental Figure 1B) or replaced by a different 
A

\begin{tabular}{|c|c|c|c|c|}
\hline & $\begin{array}{c}\text { Toll-Like } \\
\text { Receptor agonist } \\
\text { (TLRag) }\end{array}$ & $\begin{array}{l}\text { Cell-penetrating } \\
\text { peptide } \\
\text { (CPP) }\end{array}$ & $\begin{array}{l}\text { Multiantigenic } \\
\text { domain } \\
\text { (Mad) }\end{array}$ & $\begin{array}{c}\text { Toll-Like } \\
\text { Receptor agonist } \\
\text { (TLRag) }\end{array}$ \\
\hline \multirow{4}{*}{$\begin{array}{c}\text { Mouse constructs } \\
\text { (Various multiantigenic } \\
\text { domains) }\end{array}$} & & Z13 & Mad & Anaxa \\
\hline & & Z13 & Mad & \\
\hline & & & Mad & Anaxa \\
\hline & EDA & Z13 & Mad & \\
\hline $\begin{array}{l}\text { Human construct } \\
\text { (ATP125) }\end{array}$ & & Z13 & $\begin{array}{l}\text { CEA, survivin, } \\
\text { EPCAM, MUC1 }\end{array}$ & Anaxa \\
\hline
\end{tabular}

B



IL-8 (pg/ml)

D

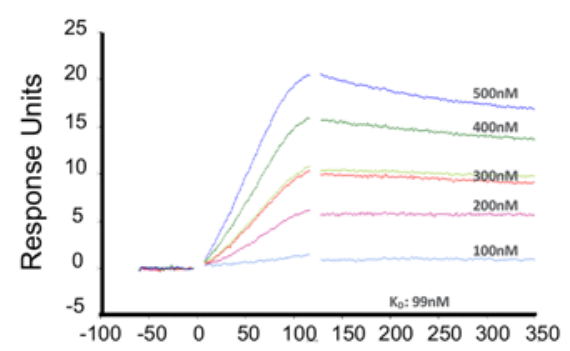

HEK-TLR4



IL-8 $(\mathrm{pg} / \mathrm{ml})$

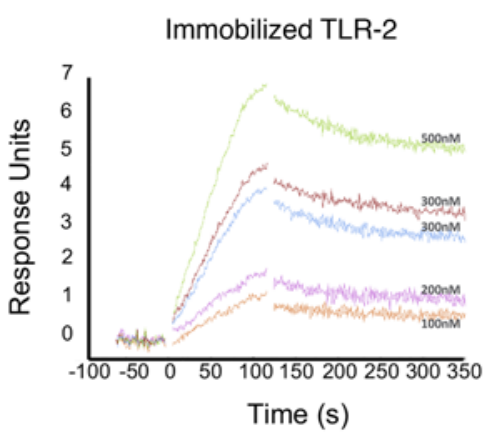

C

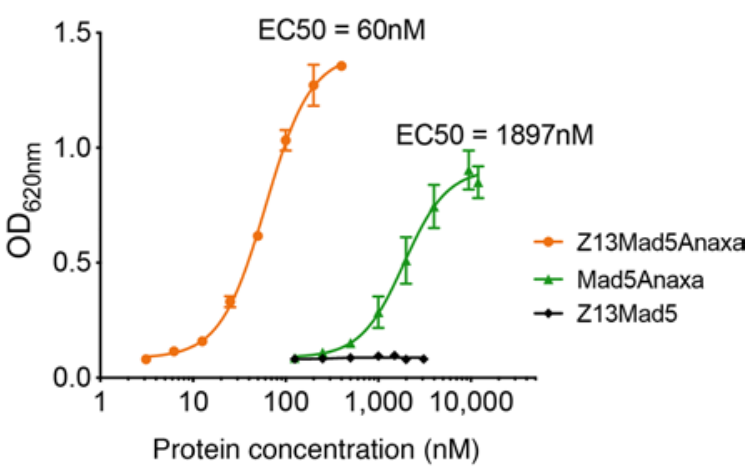

Protein concentration (nM)



Figure 1. Optimization of the vaccine construct based on self-adjuvanticity. (A) The KISIMA vaccination platform consists of 3 modules: the cell-penetrating peptide, the multiantigenic domain, and the TLR agonist. (B) HEK-Blue hTLR2 cells and HEK-Blue hTLR4 cells were incubated with $1 \mu$ M of vaccine construct or adjuvant (MPLA or Pam3Cys) medium or buffer. After 24 hours, supernatants were harvested and IL-8 measured by ELISA. One experiment shown is representative of 2 (mean $\pm \mathrm{SEM}$ ). ${ }^{*} P<0.05 ;{ }^{*} P<0.01$ (unpaired $t$ test). (C) THP1-XBlue-MD2-CD14 cells were incubated with various concentrations of vaccine constructs, medium, or buffer. After 18 hours, supernatants were recovered, and SEAP activity was measured by QUANTI-Blue assay (InvivoGen). The EC ${ }_{50}$ of the Z13Mad5Anaxa and Mad5Anaxa was calculated from the obtained dose-response curves using Prism software. (D) The binding of ATP125 to TLR4, TLR2, and TLR3 was measured by surface plasmon resonance analysis for different concentrations of ATP125: 100, 200, 300 (in duplicate), 400, and 500 nM and sensorgrams were obtained. All curves indicate the response after subtraction of nonspecific binding of molecules to a control channel.

ZEBRA-derived CPP (Z14Mad5Anaxa construct) (Supplemental Figure 1B). Concomitant administration of a TLR4 agonist (Monophosphoryl lipid A [MPLA]) or a TLR2 agonist (synthetic tripalmitoylated lipopeptide Pam3CysSerLys4 [Pam3CSK4]) with vaccine Z13Mad5 displays less efficacious antitumor activity than vaccination with the whole construct Z13Mad5Anaxa (Figure 2B and Supplemental Figure 1C).

The immunogenicity of Z13Mad5Anaxa was also compared to Z13Mad5 administered with or without the previously described adjuvants MPLA or Pam3CSK4 or to Mad5 fused to keyhole limpet hemocyanin (15). Z13Mad5Anaxa was as immunogenic as MPLA or Pam3CSK4-adjuvanted Z13Mad5 and 
A

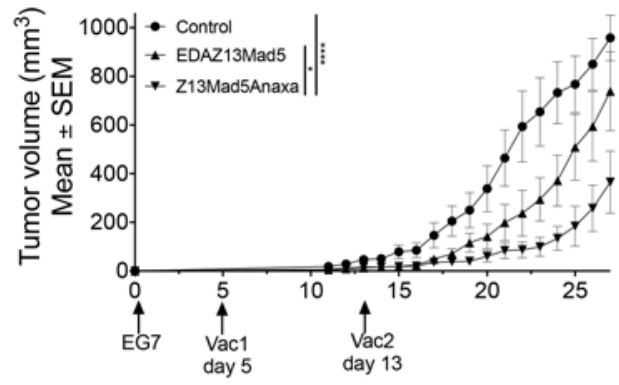

Days post-tumor implantation

\section{C}

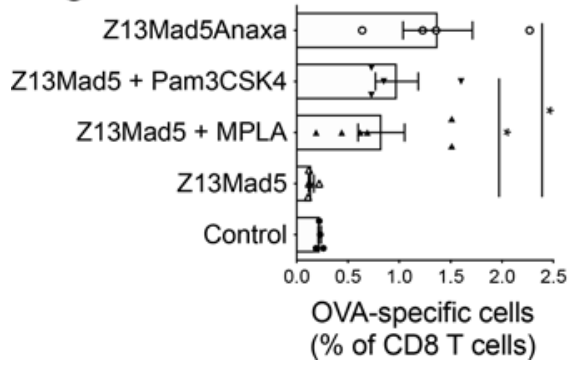

B

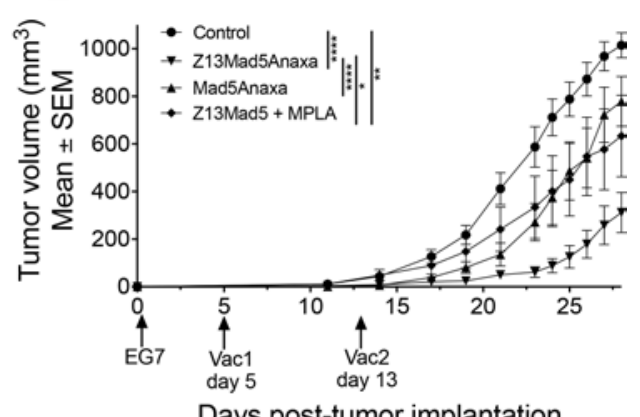

Days post-tumor implantation

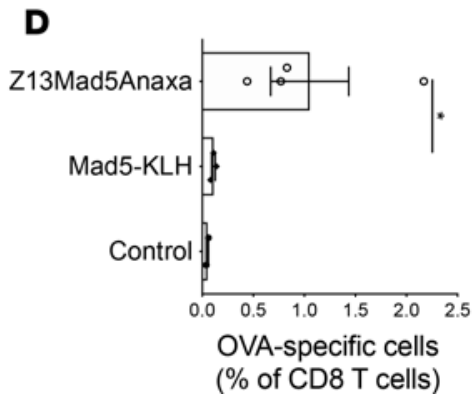

Figure 2. Z13Mad5Anaxa showed the strongest antitumor effect. (A) Tumor growth curve of C57BL/6 mice ( $n=7$ mice) group) implanted s.c. with EG7-OVA cells and vaccinated twice (day 5 and day 13) with EDAZ13Mad5 or Z13Mad5Anaxa proteins. Values are represented as the mean \pm SEM. One experiment shown is representative of $2 .{ }^{*} P<0.05 ;{ }^{* * *} P<$ 0.0001 (2-way ANOVA). (B) Tumor growth curve of C57BL/6 mice ( $n=7$ to 14 mice/group) implanted s.c. with EG7-OVA cells and vaccinated twice (day 5 and day 13) with Z13Mad5Anaxa, Mad5Anaxa, or Z13Mad5 with MPLA. Values are represented as the mean \pm SEM. A pool of 2 independent experiments is shown. ${ }^{*} P<0.05$; ${ }^{* *} P<0.01,{ }^{* * *} P<0.0001$ (2-way ANOVA). (C) Mice were vaccinated twice (day 0 and day 14) with different constructs with or without adjuvants. One week after the last vaccination, multimer staining was performed on blood cells for detecting $\mathrm{OVA}_{257-264}$-specific CD8 T cells. A pool of 3 independent experiments is shown (mean $\pm \mathrm{SEM}, n=4$ to 6 mice/group). ${ }^{*} P<0.05$ (Kruskal-Wallis test). (D) Mice were vaccinated 3 times (day 0 , day 14, day 28) with 2 different constructs. One week after the

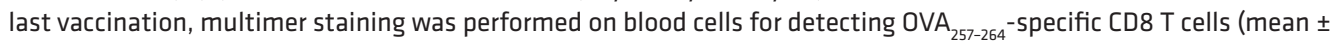
SEM, $n=2$ to 4 mice/group). ${ }^{*} P<0.05$ (Kruskal-Wallis test).

superior to nonadjuvanted (Figure 2C) or keyhole limpet hemocyanin-conjugated vaccines in terms of circulating antigen-specific CD8 T cells (Figure 2D).

Optimization of the vaccination conditions established that a vaccine dose from $2 \mathrm{nmol}$ was able to elicit a potent CD8 T cell immune response (Supplemental Figure 2A), with either synthetic peptide or recombinant protein (Supplemental Figure 2B). We identified an optimal vaccination interval of 2 weeks (Supplemental Figure 2C) and s.c. injection as the best route (Supplemental Figure 2D). A maximal immune response was observed after 3 vaccinations at 2-week intervals, with the T cell response then maintained by monthly vaccination (Supplemental Figure 2E). Multiepitopic CD8 and CD4 T cell immune responses were both elicited against different antigens: ovalbumin and self-antigen gp100 (Figure 3A), HPV antigens (Figure 3B), and glycoprotein 70 (Figure 3C) that is also a self-antigen in the BALB/c mouse strain (16). Furthermore, a significant proportion of antigen-specific CD4 and CD8 T cells are polyfunctional cells $(50 \%$ and $25 \%$, respectively), producing at least 2 different cytokines upon $\mathrm{T}$ cell receptor triggering (Supplemental Figure 2F). Interestingly, vaccination elicits circulating memory CD8 T cells, which also increased in draining lymph nodes and that mainly home and reside in the bone marrow memory compartment (Supplemental Figure 2G) with a high proportion of central memory cells within various tissues of the mice (Supplemental Figure 2H).

The self-adjuvanted vaccine platform enables the break of self-tolerance, eliciting multiepitopic effector and memory $\mathrm{CD} 8$ and $\mathrm{CD} 4 \mathrm{~T}$ cell responses, prerequisites for efficacious therapeutic cancer vaccines.

Integrated immune responses drive antitumor activity with profound modification of the tumor microenvironment. Prophylactic vaccination in the colorectal MC38 tumor model (Figure 3D) led to a 25-day improvement in median survival, which did not reach statistical significance, whereas tumor volume was significantly decreased. Additionally, therapeutic vaccination, starting 10 days after tumor cell implantation, 
A

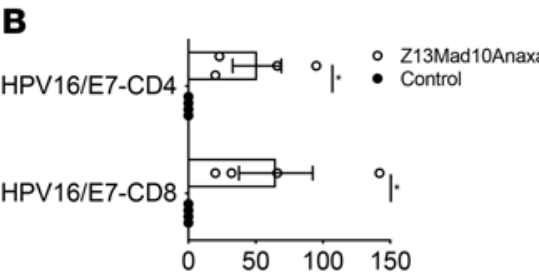

Epitope-specific spots per $10^{6}$ cells

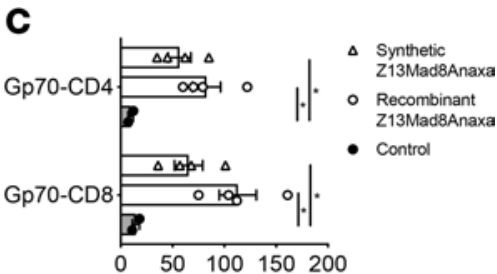

Epitope-specific spots per $10^{6}$ cells

Epitope-specific spots per $10^{6}$ cells

E

TC-1 HPV expressing fibroblasts

\section{F}

MC38 colorectal cancer

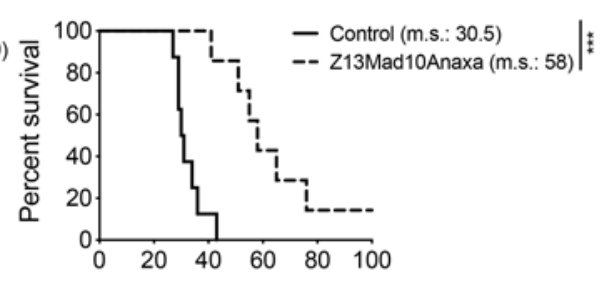

Days post-tumor implantation

Days post-tumor implantation
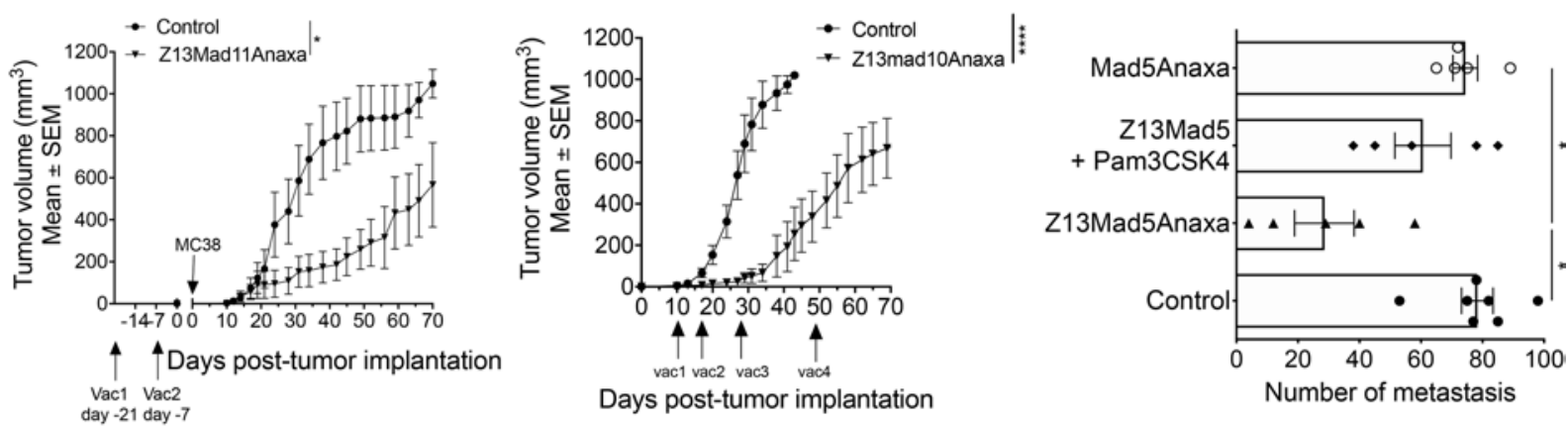

G B16-OVA lung metastasis

EG7 thymoma

MC38 colorectal cancer

TC-1 HPV expressing fibroblasts
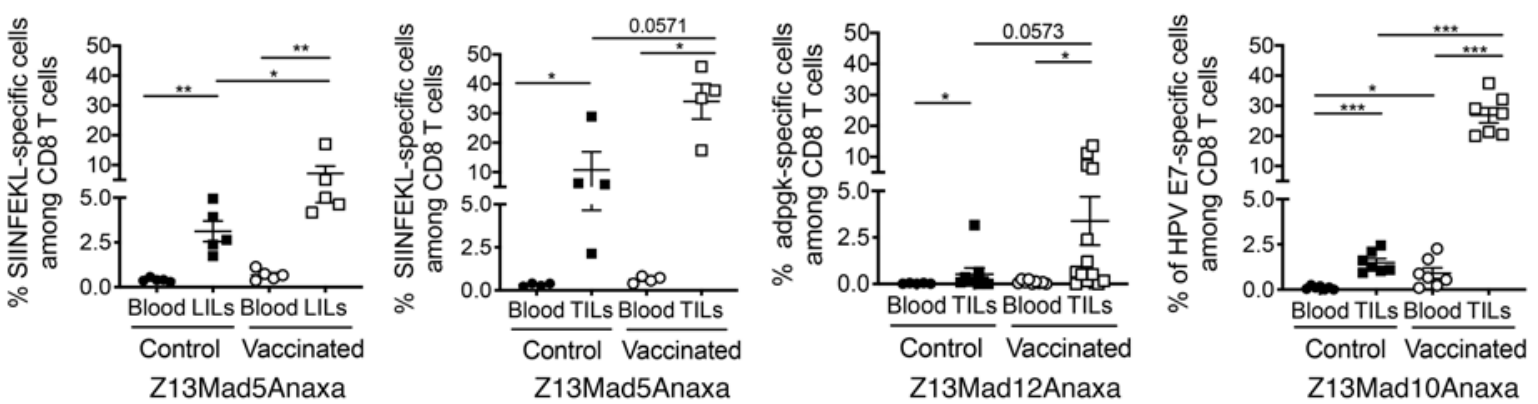

Figure 3. KISIMA vaccine induces antigen-specific T cell response in several tumor models. (A) Mice were vaccinated 6 times with Z13Mad5Anaxa, (B) 4 times with Z13Mad10Anaxa, and (C) 4 times with Z13Mad8Anaxa as a recombinant protein or synthetic peptide. One week after the last vaccination, ELISPOT assays were performed on spleen cells for detecting (A) IFN- $\gamma$-producing OVA $_{257-264}$-specific CD8 T cells (OVACD8), OVA ${ }_{323-339}$-specific CD4 T cells (OVACD4), and gp100 $25-3{ }^{-}$ specific CD8 T cells (gp100CD8). A pool of 2 independent experiments is shown ( $n=4$ to 10 mice/group). ${ }^{*} P<0.01$ (Mann-Whitney test). (B) IFN- $\gamma$-producing HPV16E7 $_{49-57}$-specific CD8 T cells and HPV16E7 ${ }_{48-57}$-specific CD4 T cells ( $n=4$ mice/group). ${ }^{*} P<0.05$ (Mann-Whitney test). (C) IFN- $\gamma$-producing gp70 ${ }_{427-435}$-specific CD8 T cells (Gp70-CD8) and gp70 ${ }_{609-622}$-specific CD4 T cells (Gp70-CD4) ( $n=2$ to 4 mice/group). ${ }^{*} P<0.05$ (Mann-Whitney test). (D) Survival curve and mean tumor volume of C57BL/6 mice ( $n=7$ mice/group) vaccinated with Z13Mad11Anaxa 21 and 7 days before s.c. implantation with MC38 cells. Median survival is indicated on the graph (m.s.). (E) Survival curve and mean tumor volume of C57BL/6 mice ( $n=7$ mice/group) implanted s.c. with TC-1 cells. Mice were vaccinated at day 10 , day 17, day 28, and day 49 with Z13Mad10Anaxa. Median survival is indicated on the graph (m.s.). One experiment shown is representative of 3 . ${ }^{*} P<0.05$, ${ }^{* * *} P<$ $0.001,{ }^{* * *} P<0.0001$ (log-rank test). (F) Mice were implanted i.v. with B16-OVA cells and vaccinated twice (day 0 and day 10) with Z13Mad5Anaxa, Mad5Anaxa, or Z13Mad5 with Pam3CSK4. On day 17, mice were euthanized and the number of lung metastatic foci was counted ( $n=5$ to 7 mice/group). A pool of 2 independent experiments is shown. ${ }^{*} P<0.05$ (Kruskal-Wallis test). (G) For different tumor models, blood cells and lung (LILs) or TILs from control and vaccinated mice were analyzed for antigen-specific CD8 T cells. C57BL/6 mice were implanted (a) i.v. with B16-OVA tumor cells, vaccinated at day 0 and day 10 and analyzed 2 weeks later ( $n=5$ mice/group); (b) s.c. with EG7 cells, vaccinated at day 5 and d13 and analyzed 1 week later ( $n=4$ mice/group); (c) s.c. with MC38 colorectal tumor cells, vaccinated at day 3, day 10, and day 17 and analyzed 1 week later (a pool of 3 independent experiments is shown [ $n=10$ to 14 mice/group]); or (d) s.c. with TC-1 tumor cells, vaccinated at day 7 and day 14 and analyzed 2 weeks later (a pool of 2 independent experiments is shown $\left[n=7\right.$ mice/group]). ${ }^{*} P<0.05$, ${ }^{* *} P<0.01$, ${ }^{* * *} P<0.001$ (Mann-Whitney test). (A-C) Mean \pm SEM are shown. 
mediated a strong antitumor effect in the tissue culture number one (TC-1) tumor model as shown by the 27-day increased median survival and significantly decreased tumor volume of vaccinated mice (Figure $3 \mathrm{E})$. Furthermore, therapeutic vaccination in the B16-OVA pulmonary metastases tumor model resulted in a significantly decreased number of metastases (Figure $3 \mathrm{~F}$ ).

An increase in proportions of antigen-specific CD8 T cells in the tumor infiltrate was observed within the B16-OVA, EG7, MC38, and TC-1 tumor models following vaccination with Z13Mad5Anaxa (B16OVA and EG7), Z13Mad12Anaxa (MC38), or Z13Mad10Anaxa (TC-1), respectively (Figure 3G). The percentage of these infiltrated cells was always significantly higher compared with the periphery in both control and vaccinated animals indicating $\mathrm{T}$ cell accumulation and/or proliferation within the tumor. The impact vaccination was the most striking in the poorly immunogenic TC-1 model where the antigen-specific CD8 T cell infiltrate was increased 30-fold in vaccinated mice compared with the control, in contrast to the 3-fold increase in the immunogenic EG7 model. The specificity of the vaccination was observed by analyzing tumor volume in the TC-1 tumor mouse model, after vaccination with either the corresponding Z13Mad10Anaxa (HPVE7 antigen) vaccine or an irrelevant Z13Mad12Anaxa (MC38 antigens) vaccine. Only the Z13Mad10Anaxa vaccination induced an antitumor response (data not shown).

The inverse correlation between the antigen-specific CD8 T cell infiltrate and the tumor size might suggest that antitumor activity relies on strong antigen-specific cancer cell elimination (Supplemental Figure 3, A and B). Alternatively, the data would also be consistent with efficient antitumor immunity occurring when tumor burden is low, revealed by production of both TNF- $\alpha$ and IFN- $\gamma$ by tumor-infiltrating antigen-specific CD 8 $\mathrm{T}$ cells (Table 1), but which is overwhelmed by the immunosuppressive microenvironment of larger tumors.

Immune suppressive cells, such as myeloid-derived suppressor cells (MDSCs), a heterogeneous population of immature myeloid cells, Tregs and tumor-associated macrophages-2 (TAM2), unlike the proinflammatory (TAM1), are a major obstacle for immunotherapy. These cells are all involved in antitumor responses by direct or indirect suppressive mechanisms inducing a tolerogenic and tumor-promoting environment $(17,18)$. In this article, we show that vaccination affected the tumor microenvironment (TME) by decreasing the immunosuppressive granulocytic MDSCs in the EG7 model resulting in an increased proportion of functional antigen-specific CD8 T cells producing IFN- $\gamma$ and TNF- $\alpha$. Similarly, in the TC- 1 tumor model, we observed an enhanced proportion of functional antigen-specific CD8 T cells and a favorable TME characterized by improved CD8/Treg and the TAM-type 1/type 2 ratios (Table 1). These results indicate that this potentially new vaccine increases the tumor infiltration by antigen-specific $\mathrm{T}$ cells and can favorably modulate a protumoral microenvironment resulting in diminished tumor size.

Vaccination generates a humoral immune response that does not affect efficacy of subsequent vaccinations. A humoral response was detected in mice only after the third vaccination with Z13Mad5Anaxa, with increasing titers following each subsequent vaccination (Supplemental Figure 4A). An increased proportion of OVA $_{257-264}$-specific CD8 T cells in the blood was still observed after the fourth, fifth, and sixth vaccinations despite the accumulation of anti-vaccine antibodies in the serum (Supplemental Figure 4B), with no inverse correlation between $\mathrm{T}$ cell and antibody responses (Supplemental Figure 4C). To confirm that anti-vaccine antibodies do not interfere with vaccine activity, Z13Mad5Anaxa was preincubated with serum from Z13Mad5Anaxa-immunized mice before vaccination. After 2 vaccinations, we observed a comparable proportion of $\mathrm{OVA}_{257-264}$-specific CD8 T cells in the blood of mice vaccinated with Z13Mad5Anaxa preincubated with serum from either vaccinated or naive mice (Supplemental Figure 4D). These results indicate that a humoral response against the vaccine does not alter the potency of the induced $\mathrm{T}$ cell response.

Validation of a new construct encompassing human antigens. The ATP125 vaccine was designed with human sequences of TAA described in colorectal cancer (CRC) (19-22), with a Mad composed of portions of CEA, MUC1, EPCAM, and survivin antigens (Supplemental Table 1) and comprising Z13 and Anaxa domains. Blood-derived human DCs were exposed in vitro to the ATP125 vaccine and assessed by flow cytometry for extracellular or intracellular staining via the use of an anti-His antibody detecting ATP125. Figure 4A shows an increase of both extracellular and intracellular staining of ATP125 with time, demonstrating its surface exposition and its penetration inside the cells.

Natural processing and presentation of ATP125 on HLA molecules by human DCs from 6 healthy donors was evaluated using mass spectrometry-based HLA ligandome analysis and mapping after overnight loading (Figure 4B and Tables 2 and 3). For each donor, both class I- and class II-restricted ATP125 Mad-derived peptides were presented by DCs (Supplemental Tables 2 and 3). HLA-binding motifs predicted with the NetMHCpan 3.0 and SYFPEITHI algorithms were also identified. Peptides derived from all the antigens 
Table 1. Tumor size and tumor-infiltrating leucocytes

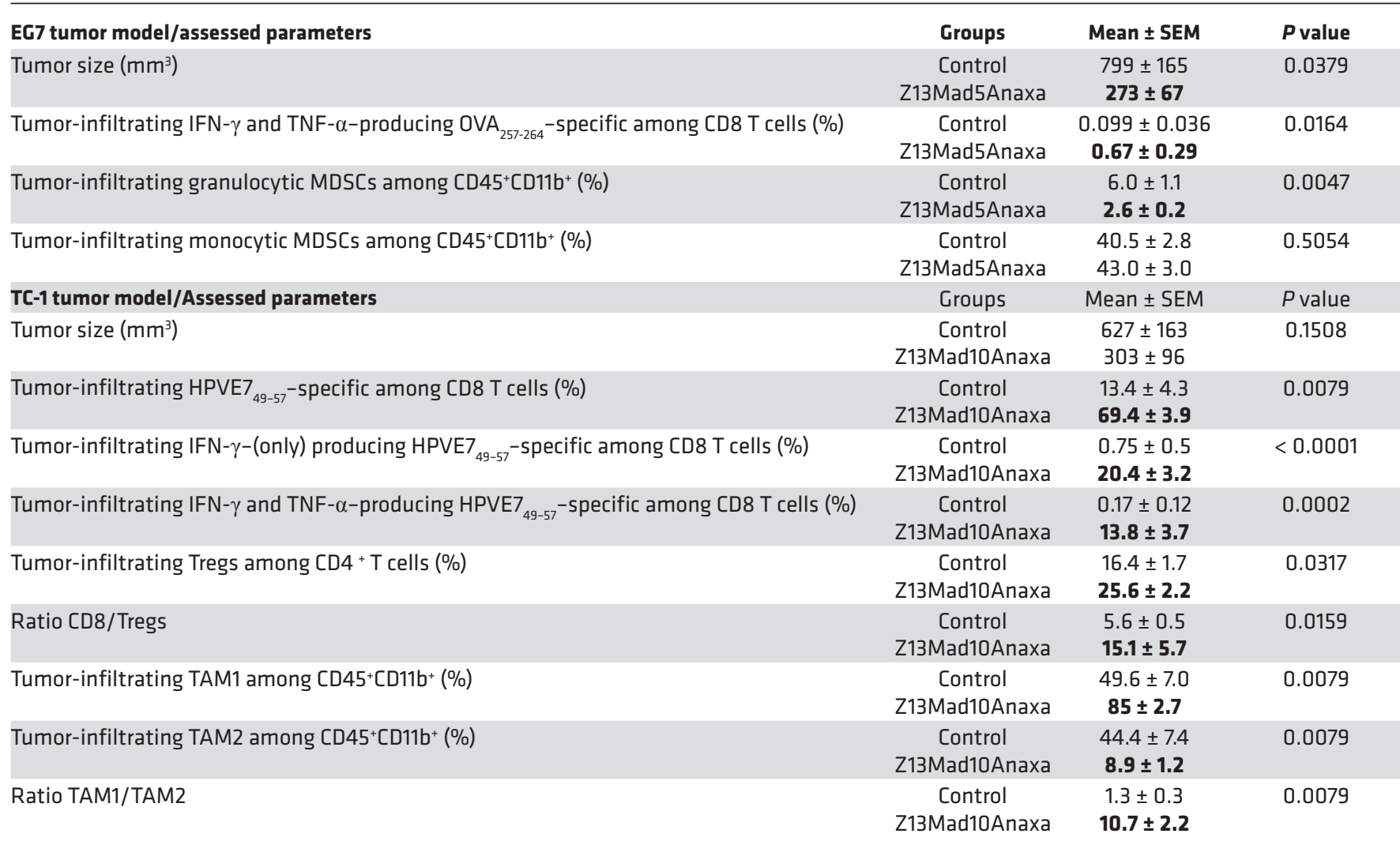

Bold indicates statistical significance

except MUC1 were identified to be presented on HLA class I molecules (Tables 2 and 3). The junction of the EPCAM antigen and Anaxa created some class I neoepitopes of 8-12 amino acids in 5 of 6 donors (Supplemental Table 2). Homology analysis of the spanning region of 17 residues from which the various neoepitopes originated, showed that this promiscuously HLA-binding neoepitope has $75 \%$ identity with the ComEC/Rec2-related protein and $73 \%$ identity with the S-phase kinase-associated protein 2 . A higher number of class II-restricted peptides were identified from all the antigen portions of the vaccine: 6 of 6 donors presented HLA class II epitopes from CEA and survivin, 5 of 6 from EPCAM, and 3 of 6 from MUC1 (Tables 2 and 3). The ligandome analysis showed that more than 4,000 different class I and 5,800 class II peptides were presented as an average per donor by DCs, corresponding to 2,700 and 1,250 different proteins, respectively. After subtracting protein found for MPLA-activated DCs, we identified 94 proteins from which derived peptides were presented by at least 3 donors for the ATP125-loaded condition; these were not presented by nonloaded MPLA-activated DCs. To determine whether ATP125-mediated activation can be linked to specific biological processes, Gene Ontology term enrichment analysis was performed on this set of 94 proteins and revealed processes mainly related to the regulation of the adaptive immune response (Supplemental Figure 5).

In vitro, ATP125 activated blood-derived human DCs, based on analysis of DC-specific activation marker overexpression: HLA-DR, CD80, CD83, and CD86 (Figure 4C). The cytokine/chemokine secretion signature of ATP125-loaded DCs or peripheral blood mononuclear cells (PBMCs) was compared to TLR2 (Pam3CSK4), TLR4 (MPLA), and TLR7/8 (R848) ligand-loaded DCs and revealed similarity to that of MPLA with secretion of IL-6, IL-8, IL-12p70, TNF- $\alpha$, IP-10, MIP-1 $\alpha$, and MIP-1 $\beta$ by human monocyte-derived DCs (moDCs) and secretion of IL-6, IL-8, TNF- $\alpha$, MIP-1 $\alpha$, and MIP-1 $\beta$ by PBMCs (Figure 4D).

Vaccinations are safe in NHPs. Cynomolgus monkeys received s.c. or intradermal (i.d.) injection of ATP125 at 2 dose levels ( $76 \mu \mathrm{g}$ or $380 \mu \mathrm{g}$ /vaccination, corresponding to 2 and $10 \mathrm{nmol}$, respectively), every 2 weeks for a total of 4 vaccinations in a toxicology study (Figure 5A). The 4 vaccinations were well tolerated, and no significant outcome was observed on body weight, food consumption, or hematological and 
A

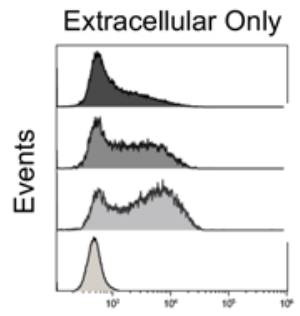

Anti $-6 \mathrm{His}$

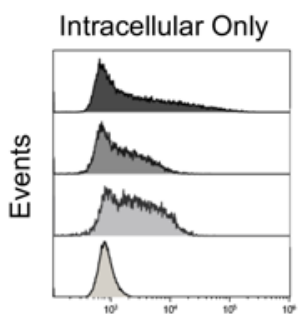

Anti $-6 \mathrm{His}$



Anti $-6 \mathrm{His}$

B Class I epitopes

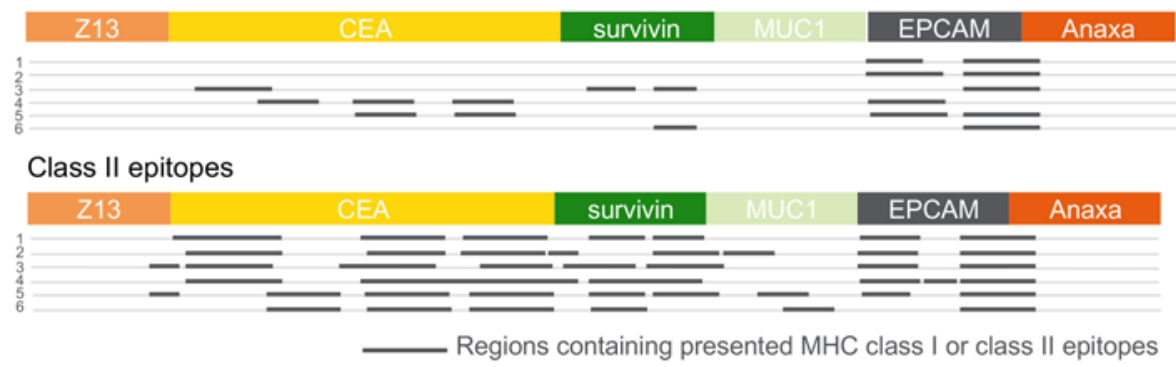

C

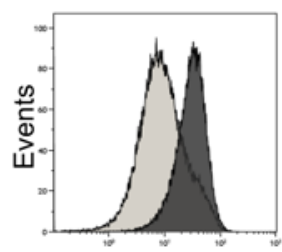

HLA-DR

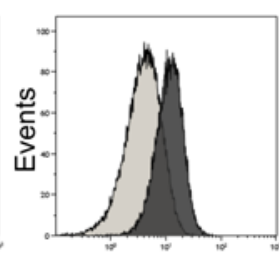

$\mathrm{CD} 80$

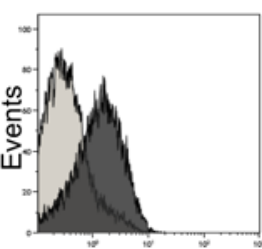

CD83

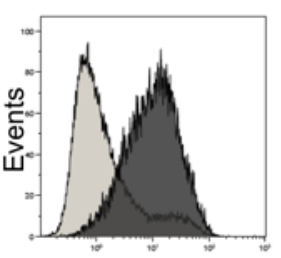

CD86
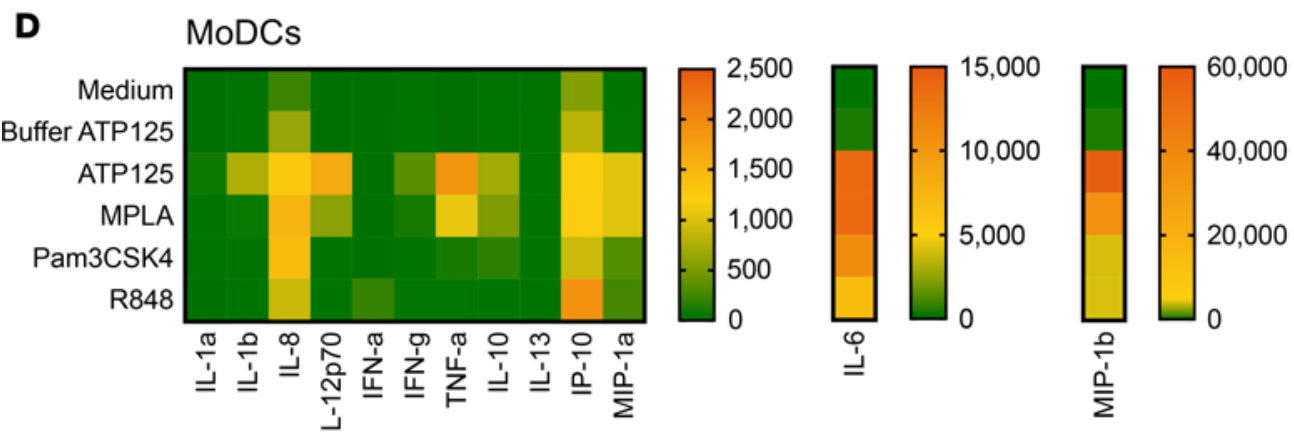

PBMCs
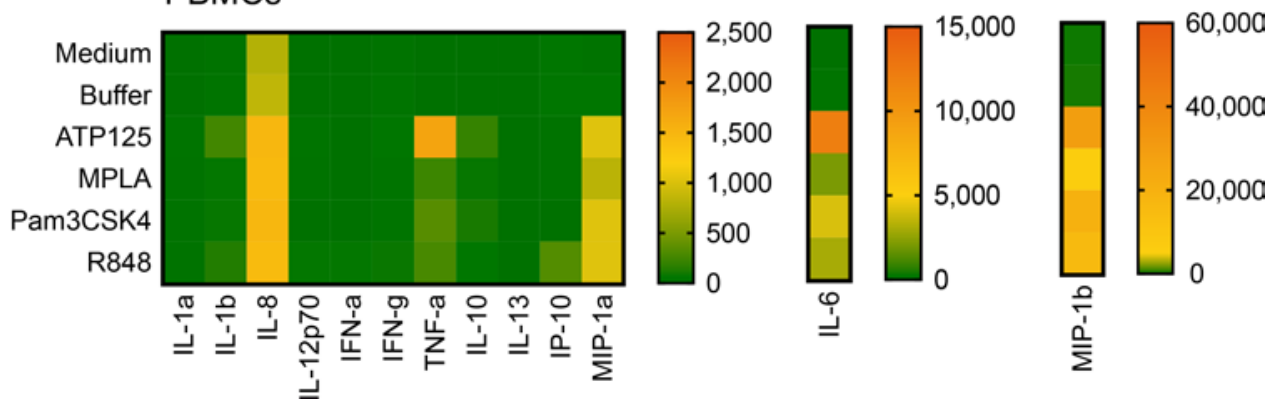

Figure 4. ATP125 is processed and presented by human DCs. (A) Representative intracellular and extracellular anti-His staining of human DCs stimulated with $300 \mathrm{nM}$ of ATP125. One experiment shown is representative of 3. (B) Identification of naturally presented HLA class I- and HLA class II-restricted peptides by ATP125-loaded human DCs using a mass spectrometry-based immunopeptidomics approach. The bold lines indicate regions of the recombinant protein containing HLA class I- or class II-presented peptides. (C) Human DCs were cultured overnight with $300 \mathrm{nM}$ of ATP125 before cytometry analysis. Histograms of activation markers are shown. One experiment shown is representative of 3. (D) Heatmap of secreted cytokines/chemokines $(\mathrm{pg} / \mathrm{ml})$ by 24-hour activated human DCs $(n=3)$ or human PBMCs $(n=$ 3) with $300 \mathrm{nM}$ of ATP125, MPLA (TLR4 ligand), Pam3CSK4 (TLR2 ligand), or R848 (TLR7/8 ligand), equivalent volume of ATP125 buffer and control cells. 
Table 2. HLA haplotype for each donor and total number of ATP125-derived HLA class I-presented peptides

\begin{tabular}{cccccc}
\hline Donor & HLA class I haplotype & CEA & survivin & MUC1 & EPCAM \\
1 & $A^{*} 03, A^{*} 23, B^{*} 15: 03 / 103 / 220, B^{*} 44$ & 0 & 0 & 0 & 0 \\
2 & $A^{*} 02: 01, A^{*} 02: 05, B^{*} 40: 05, B^{*} 44$ & 0 & 0 & 0 & 1 \\
3 & $A^{*} 02: 02, A^{*} 34: 02, B^{*} 18, B^{*} 44$ & 1 & 2 & 0 & 0 \\
4 & $A^{*} 02: 01, A^{*} 02: 06, B^{*} 35, B^{*} 48$ & $4-1$ & 0 & 0 & 4 \\
5 & $A^{*} 02: 01, A^{*} 29, B^{*} 08, B^{*} 40$ & 3 & $\mathbf{1}$ & 0 & 0
\end{tabular}

The number of peptides showing a canonical HLA-binding motif, and thereby predicted by NetMHCpan 3.0 or SYFPEIHI as HLA class I ligands, is indicated in bold.

biochemical parameters (data not shown). Very slight to well-defined erythema was observed on the day after each vaccination for all groups but spontaneously resolved. No dose-dependent changes in cytokine release or leukocyte subpopulation proportions were observed (Supplemental Figure 6A and 6B). Necropsy of treated animals indicated that organ weights were within the normal range for animals of this age, weight, and sex. Microscopic examination of spleen and lymph node samples did not show any significant morphologic differences between animals from the 4 groups (data not shown).

Vaccinations elicit antigen-specific $T$ cell responses and humoral responses in NHPs. Immunized cynomolgus macaques were assessed for antigen-specific immune responses elicited by the vaccine in the peripheral blood. For this purpose, we used 3 complementary methodologies: T cell intracellular cytokine staining, IFN- $\gamma$ enzyme-linked immunospot (ELISPOT), and $\mathrm{T}$ cell proliferation performed after short-, mid- or long-term peptide activation. T cell immune responses were detected in fresh PBMCs for all groups of animals 7 days after the last vaccination (Figure 5B, Table 4, and Supplemental Figure 6, A and B). However, whereas all 6 animals vaccinated by the s.c. route elicited an immune response against the 4 antigens, 4 of 6 i.d. vaccinated animals showed an immune response against the 4 antigens (Supplemental Figure 6C). Furthermore, a multiantigenic CD8 T cell response was preferentially observed in the high-dose group for the s.c. route with 3 of 3 animals mounting an immune response against at least 3 antigens, detected by intracellular cytokine staining. Similarly, ELISPOT assays highlighted a multiantigenic response in 2 of 3 animals against all 4 antigens. Overall, these data showed a preferential multiantigenic T cell immune response in the group vaccinated with the high dose of $380 \mu \mathrm{g}(10 \mathrm{nmol})$ of ATP125 by the s.c. route.

A humoral immune response was preferentially detected in animals vaccinated by the s.c. route, demonstrated by higher anti-ATP125 and anti-survivin IgG titers compared with the i.d. route groups (Supplemental Figure 6, D and E).

Significant additive effect of self-adjuvanted vaccine in combination with programmed cell death protein 1 blockade on tumor growth. The potential of anti-programmed cell death protein 1 (anti-PD1) antibody combination was investigated in the MC38 CRC model in which vaccine alone showed a low therapeutic efficacy (Figure 6A) and in the TC-1 lung cancer model (Figure 6B). Whereas the anti-PD1 alone showed significant efficacy in the MC38 tumor model compared with controls, the combination of both vaccine and PD1 blockade resulted in a highly significant additive effect with 10 of 14 mice tumor-free, accompanied by a significant decreased tumor volume (Figure 6A). In the TC-1 tumor model, treatment with anti-PD1 alone did not show efficacy in contrast

Table 3. HLA haplotype for each donor and total number of ATP125-derived HLA class II-presented peptides

\begin{tabular}{|c|c|c|c|c|c|}
\hline Donor & HLA class II haplotype & CEA & survivin & MUC1 & EPCAM \\
\hline 1 & $\mathrm{DRB}^{*} 04, \mathrm{DRB}^{*} 07, \mathrm{DQB} 1^{*} 02: 02, \mathrm{DQB} 1^{*} 03$ & 46 & 3 & 0 & 5 \\
\hline 2 & $\mathrm{DRB}^{*} 03, \mathrm{DRB} 1^{*} 04, \mathrm{DQB} 1^{*} 02, \mathrm{DQB} 1^{*} 03$ & 78 & 5 & 1 & 5 \\
\hline 3 & $\mathrm{DRB}^{*} 14, \mathrm{DRB} 1^{*} 15: 03, \mathrm{DQB} 1^{*} 05, \mathrm{DQB} 1^{*} 06$ & 23 & 22 & 0 & 2 \\
\hline 4 & DRB1*04:04, DRB1*04:07, DQB1*03:02 & 93 & 10 & 0 & 9 \\
\hline 5 & $\mathrm{DRB1}^{*} 03: 01, \mathrm{DRB} 1^{*} 07, \mathrm{DQB1}{ }^{*} 02: 01, \mathrm{DQB} 1^{*} 02: 02$ & 10 & 9 & 4 & 1 \\
\hline 6 & $\mathrm{DRB1}^{*} 07, \mathrm{DRB1}{ }^{*} 15, \mathrm{DQB1}{ }^{*} 02: 01, \mathrm{DQB1}{ }^{*} 06: 02$ & 19 & 18 & 2 & 0 \\
\hline
\end{tabular}


A

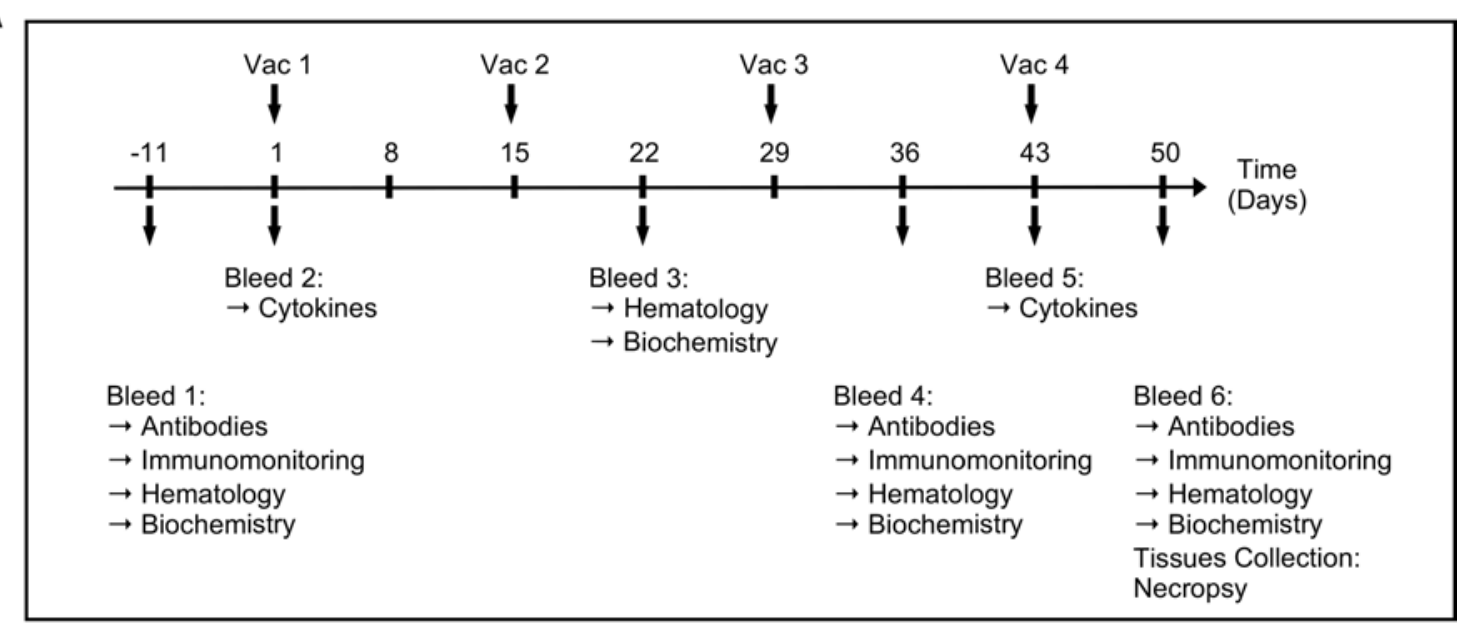

B

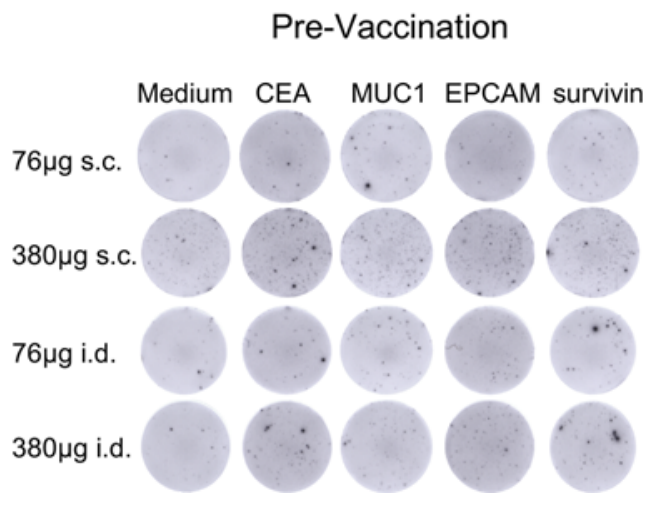

Post Vaccination Day 50

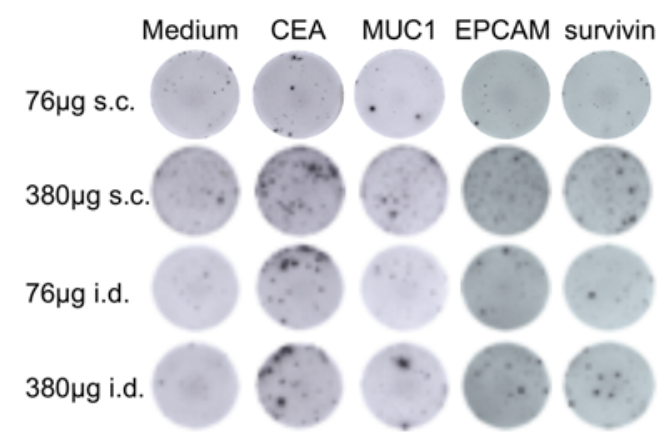

Figure 5. ATP125 elicits T cell responses in NHPs. (A) Vaccination and assessment schedule of Cynomolgus fascicularis NHPs. (B) Cellular immune response against CEA, MUC-1, survivin, and EPCAM was monitored by IFN- $\gamma$ ELISPOT on PBMC prior to any vaccination (left panel) or 1 week after the last vaccination on day 50 (left panel) in animals vaccinated with 76 or $380 \mu \mathrm{g}$ of ATP125 s.c. or i.d. Representative ELISPOT IFN- $\gamma$ wells for 1 animal of 3 for each treatment group are shown.

to vaccine alone, which led to improved median survival and significantly decreased tumor volume. Combinatory treatment significantly improved efficacy as shown by the 31- and 14-day increased median survival in comparison with single treatment with anti-PD1 or vaccine, respectively and also characterized by significantly decreased tumor volume. These encouraging preliminary data show that the combination is a highly efficacious therapy, and further investigations are required to evaluate tumor infiltration and TME modulation.

\section{Discussion}

With the combined effect of a CPP and a TLR2/4 peptide agonist for the initiation of an efficacious multiantigenic $\mathrm{T}$ cell response, the vaccination strategy described herein addresses several challenges faced by therapeutic cancer vaccines. These challenges include the need to overcome the heterogeneity of individual tumors $(3,4)$, to trigger tumor infiltration (6), and to elicit CD8 and CD4 $\mathrm{T}$ cell immune responses facilitating eradication of tumoral cells and relapse prevention $(23,24)$.

The first key finding was the self-adjuvanticity of the vaccine platform, which did not rely on an exogenously administered adjuvant. All DCs presenting vaccine-derived epitopes were activated through the TLR-2/4 pathways and upregulated costimulatory molecule expression as previously described for the annexin II protein $(25,26)$. DCs loaded with self-adjuvanted vaccine secreted MIP1- $\alpha$, MIP1- $\beta$, IP10, and IL-12p70 that play a crucial role in T cell recruitment, Th1 response induction, and IFN- $\gamma$ secretion necessary for antitumor immunity (27). Moreover, in vitro, we observed a significant increase of DC activation when both the CPP and the Anaxa were on the same molecule. This finding could be a result of longer exposure of the Anaxa to TLR molecules due to CPP binding and/or intracellular activation of TLR4 (28) due to CPP-mediated internalization; further investigation is required to determine the relative importance 
Table 4. Spots/million of blood CD3+ cells from NHP vaccinated with 76 or $380 \mu$ of ATP125 s.c. or i.d.

\begin{tabular}{|c|c|c|c|c|c|c|c|c|c|}
\hline & Stimulant & CEA & & MUC1 & & EPCAM & & Survivin & \\
\hline \multirow{2}{*}{$76 \mu \mathrm{g} /$ animal s.c. } & 1 & 3 & 9 & 23 & 0 & 0 & 0 & 2 & 0 \\
\hline & 3 & 0 & 43 & 0 & 0 & 0 & 50 & 2 & 24 \\
\hline \multirow[b]{2}{*}{$380 \mu \mathrm{g} /$ animal s.c. } & 1 & 2 & 84 & 0 & 24 & 0 & 106 & 0 & 25 \\
\hline & 2 & 0 & 89 & 0 & 58 & 0 & 84 & 0 & 145 \\
\hline \multirow[t]{2}{*}{$76 \mu \mathrm{g} / a n i m a l$ i.d. } & 2 & 0 & 0 & 0 & 6 & 0 & 30 & 0 & 42 \\
\hline & 3 & 0 & 104 & 0 & 30 & 0 & 34 & 0 & 72 \\
\hline \multirow{3}{*}{$380 \mu \mathrm{g} / a n i m a l$ i.d } & 1 & 0 & 7 & 0 & 0 & 0 & 0 & 0 & 0 \\
\hline & 2 & 20 & 205 & 0 & 24 & 24 & 63 & 21 & 64 \\
\hline & 3 & 0 & 40 & 0 & 57 & 0 & 0 & 0 & 0 \\
\hline
\end{tabular}

Vac, vaccination; vac4, 4th vaccination.

of these processes. In vivo, vaccine efficacy was directly linked to the presence of the CPP and the TLRag in 1 single protein because when modules are separated, no therapeutic benefit in any tumor models was observed. Although adjuvant-conjugated vaccines may also be able to activate every DC loaded with the antigen (29), the CPP element brings the additional advantages of intracellular delivery.

In addition, it was consistently shown, for various multiantigenic constructs, that CPP-mediated antigen uptake, not only with external adjuvant $(10,11)$ but also within a self-adjuvanting protein, can promote CD8 and CD4 T cell immune responses. Furthermore, these immune responses were not limited to model antigens or neoantigens but were also induced for self-antigens both in mice (gp100 and gp70) and in NHPs (CEA, survivin, EPCAM, and MUC1). This led to efficacious antitumor responses as shown in MC38 mouse models upon vaccination against survivin. This was linked to induction of antigen-specific $\mathrm{T}$ cells, as vaccination with a nonrelevant antigen was ineffective (data not shown). The correct processing and presentation of multiantigenic cargos by human DCs was assessed by analyzing the ligandome on different donors. As in mice and in NHPs, we showed that epitopes were presented on both MHC class I and II molecules. The DC ligandome analysis performed on multiple donors with different HLA haplotypes confirmed the multiallelic binding properties of the vaccine antigens and also revealed creation of a neoepitope at the junction between Anaxa and EPCAM, highlighting the interest and strength of using mass spectrometry-based ligandome analysis in the design of chimeric multiantigenic proteins.

Self-adjuvanticity is a key feature of viral or bacterial vectors. However, neutralizing antibodies very often limit the number of administrations to a single injection (30), and clinical development must take into account the essential multiple safety measures necessary for live recombinant vectors. In contrast, humoral responses elicited by the inert vaccine do not negatively affect $\mathrm{T}$ cell-mediated immune responses. Moreover, this humoral immune response is expected to boost $\mathrm{T}$ cell response, as formation of immune complexes improves both antigen uptake and consequently CD8 T cell recall responses $(31,32)$.

The initial study performed in NHPs indicated a good safety profile, with no toxicity observed. Overall, we introduced a class of self-adjuvanting vaccines, possessing a versatile cargo. This platform could be developed either as a synthetic long peptide for personalized vaccination or as a recombinant multiantigenic vaccine giving rise to responses restricted by multiple HLA alleles as a standardized vaccine approach.

Finally, significant changes in the TME have been observed upon vaccination, including (a) massive tumor infiltration of antigen-specific effector T cells, (b) reduction of MDSCs and Tregs, and (c) M1 polarization of macrophages. Moreover, combination with PD1 blockade was shown to have an additive effect and to significantly increase the efficacy of our vaccine in cancer models. Altogether, these data indicate that this vaccination strategy is an ideal partner to combine with checkpoint inhibitors. It has been observed that the tumors of patients resistant to PD1 blockade have limited immune infiltration $(33,34)$. Therefore, identifying therapies, such the vaccination strategy presented herein, which strikingly enhances $\mathrm{T}$ cell infiltration, could render tumors sensitive to checkpoint inhibitors, one of the current major challenges in cancer immunotherapy. 
A



B

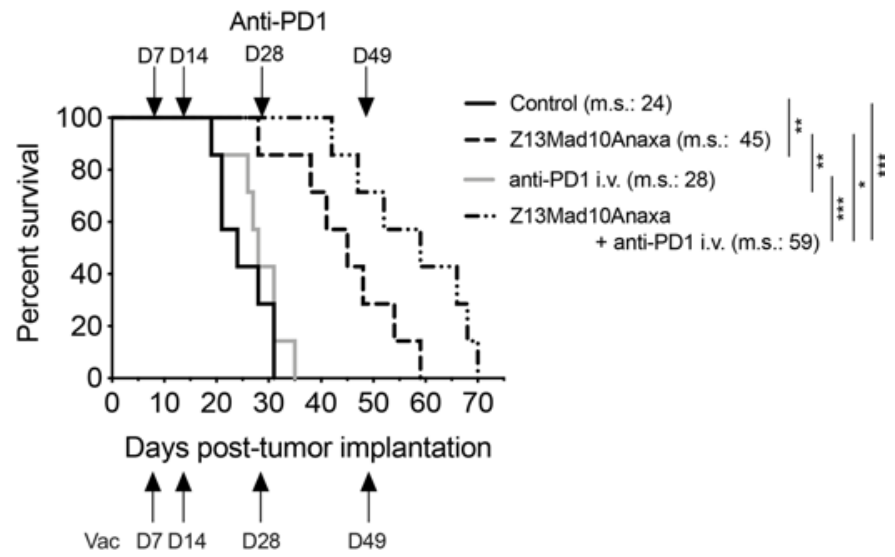

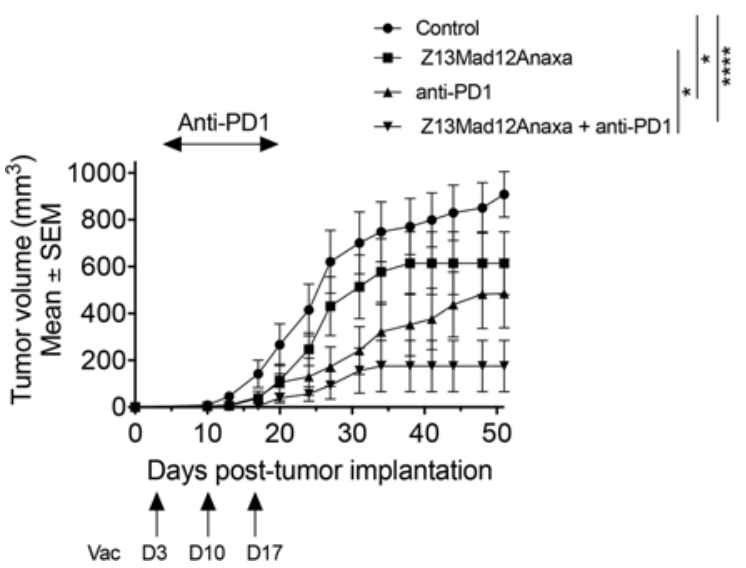

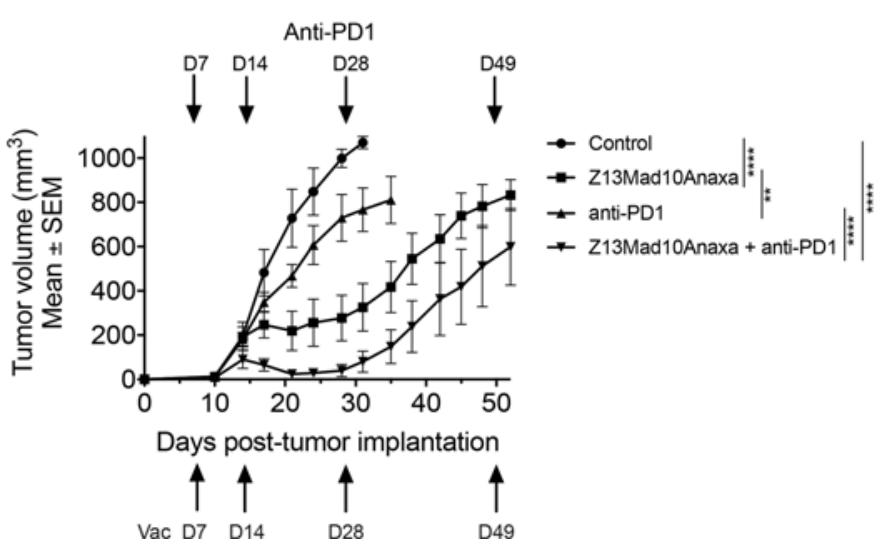

Figure 6. Self-adjuvanting efficacy of KISIMA vaccines further enhanced with anti-PD1 therapy. (A) Survival curve and mean tumor volume of C57BL/6 mice ( $n=14$ mice/group) implanted s.c. with MC38 cells. One group was used as control. In the other groups, mice were vaccinated with Z13Mad12Anaxa s.c. on day 3, day 10, and day 17, injected with a mouse-specific anti-PD1 i.p. from day 6 to day 31, or received the combination treatment. A pool of 2 independent experiments is shown. (B) Survival curve and mean tumor volume of C57BL/6 mice ( $n=7$ mice/group) implanted s.c. with TC-1 cells. One group was used as control. In the other groups, mice were vaccinated with Z13Mad10Anaxa s.c. on day 7, day 14, day 28, and day 49, injected with a mouse-specific anti-PD1 i.v. on the same days, day 7, day 14, day 28, and day 4, or received the combination treatment. One experiment shown is representative of 3. Median survival is indicated on the survival graph (m.s.). ${ }^{*} P<0.05$; ${ }^{* *} P<0.01 ;{ }^{* * *} P<0.001,{ }^{* * * *} P<0.0001$ (log-rank test). ${ }^{*} P<0.05$, ${ }^{* *} P<0.01,{ }^{* * * *} P<$ 0.0001 (2-way ANOVA for tumor growth curves).

\section{Methods}

Animals. Female C57BL/6J and BALB/cJ mice were purchased from Charles River Laboratories. All mice used in this study were females between 6 and 12 weeks of age at the time of experiments. Male cynomolgus monkeys (Macaca fascicularis) were purchased from Le Tamarinier, enrolled in a study conducted by CiToxLab, and were between 34 and 35 months of age at the time of the study.

Vaccines. Vaccine constructs were designed in-house and produced in E. coli by Genscript. Vaccines were prepared by dilution in vaccine buffer. In some experiments, synthetic peptides synthetized by Pepscan were used. Vaccinations were mainly performed s.c. ( $2 \mathrm{nmol})$. However, 0.5 , 2, or $10 \mathrm{nmol}$ of vaccine was used in some experiments. In addition, different routes were also assessed, for example - i.v., i.m., intranodal, or i.d. During the purification process, endotoxins were removed from vaccines through extensive washes with Triton-X114 followed by subsequent affinity chromatography. Endotoxin content was quantified in each vaccine batch using a LAL chromogenic assay. Only the batches with an endotoxin level less than or equal to 10 EU/ $\mathrm{mg}$ protein (according to guidelines) were used for further in vitro/in vivo experiments.

Cell lines. The EG.7-OVA cell line (EG7, CRL-2113; ATCC), a stable transfectant of the murine OVA-expressing EL-4 thymoma (H-2 ${ }^{b}$ ), was maintained in complete RPMI 1640 medium with $0.4 \mathrm{mg} / \mathrm{ml}$ geneticin (Life Technologies). The B16-OVA cell line (provided by Bertrand Huard, University of Grenoble-Alpes, Grenoble, France), an OVA-transfected clone derived from the murine melanoma cell line B16 was maintained in 
complete RPMI 1640 medium with $1 \mathrm{mg} / \mathrm{ml}$ geneticin. The TC-1 cell line (The Johns Hopkins University), a lung epithelial cell line with HPV16 E6 and E7 and c-H-ras oncogenes was maintained in complete RPMI 1640 with $0.4 \mathrm{mg} / \mathrm{ml}$ geneticin. The MC38 cell line (provided by Bertrand Huard, University of Grenoble-Alpes), a murine CRC cell line, was maintained in complete DMEM containing 5\% of gentamicin. The HEK-Blue hTLR2 and HEK-Blue hTLR4 cell lines (InvivoGen) were maintained in DMEM Glutamax with $100 \mu \mathrm{g} / \mathrm{ml}$ of Normocin (InvivoGen). Medium was supplemented with HEK-Blue selection $\times 1$ (InvivoGen) after cells had been passaged twice. THP1-XBlue-MD2-CD14 cells (InvivoGen) were maintained in RPMI 1640 Glutamax with 25-mM HEPES (Gibco) and $100 \mu \mathrm{g} / \mathrm{ml}$ of Normocin (InvivoGen). Medium was supplemented with 200 $\mu \mathrm{g} / \mathrm{ml}$ of Zeocin and $250 \mu \mathrm{g} / \mathrm{mL}$ of G418 (InvivoGen) after cells were passaged twice.

In vivo tumor experiments. C57BL/ 6 mice were implanted s.c. with $3 \times 10^{5}$ EG7-OVA tumor cells in the left flank and vaccinated twice (day 5 and day 13) with a 2-nmol s.c. vaccine injection in the right flank. Tumor size was measured with a caliper. Mice were euthanized when their tumor reached a diameter of $13 \mathrm{~mm}$. For tumor-infiltrating lymphocytes (TILs), tumors were harvested, chopped, digested in collagenase D/DNAse enzyme mix for 30 minutes at $37^{\circ} \mathrm{C}$, and filtered through a $70-\mu \mathrm{m}$ cell strainer. TILs were then purified by Ficoll separation.

C57BL/ 6 mice were implanted i.v. with $1 \times 10^{5}$ B16-OVA tumor cells and vaccinated twice (day 0 and day 10) with a 2-nmol s.c. vaccine injection. At day 17 after implantation, lungs were perfused with Ringer solution, recovered, and metastases were counted using microscopy.

C57BL/ 6 mice were implanted s.c. with $1 \times 10^{5}$ TC- 1 tumor cells in the back and vaccinated twice (day 10 and day 17) with a 2-nmol s.c. vaccine injection at the tail base. Tumor size was measured with a caliper. Mice were euthanized when the tumor reached a diameter of $13 \mathrm{~mm}$.

C57BL/ 6 mice were implanted s.c. with $2 \times 10^{5} \mathrm{MC} 38$ tumor cells in the back. Vaccination schedule was either prophylactic ( 3 weeks and 1 week before tumor implantation) or therapeutic (day 3, day 10, and day 17 after tumor implantation) with a 2 -nmol s.c. vaccine injection at the tail base. Tumor size was measured with a caliper. Mice were euthanized when tumor reached a diameter of $13 \mathrm{~mm}$.

In vivo NHP toxicological study. Cynomolgus monkeys received 4 injections of ATP125 every 2 weeks via the s.c. or i.d. route at 2 levels of dosage: $76 \mu \mathrm{g}$ or $380 \mu \mathrm{g}$ /vaccination, corresponding to 2 and $10 \mathrm{nmol}$, respectively. Local reactions at the injection sites were evaluated before each administration, then 24,48 , and 72 hours after injection. Local reaction recording was prolonged until recovery when findings were present. The animals were checked twice daily for clinical signs. Rectal temperature was recorded before each vaccination and 1, 8, and 24 hours after vaccination. Body weight was recorded twice before the beginning of the treatment period, on the first 3 days of the study, and at least once a week until the end of the study. Food consumption was checked daily.

Hematology and blood biochemistry investigations were performed before the beginning of the treatment period, on day 22, and at the end of the study (day 50). Blood collections were performed for antiATP125 antibody titration and immune response evaluation before the first vaccination and on days 22,36 , and 50. Determination of cytokine blood levels was performed once before the beginning of the treatment period, before vaccination (on day 43 only), and then 1, 4, and 8 hours after vaccination on days 1 and 43 .

On completion of the treatment period, the animals were euthanized, and a full macroscopic postmortem examination was performed. Brain, cecum, colon, duodenum, heart, ileum, jejunum, kidneys, liver, lungs with bronchi, inguinal and mesenteric lymph nodes, spleen, testes, thymus, and injection sites were weighed and selected tissues were preserved. A microscopic examination was performed on injection sites, inguinal lymph nodes, and spleen from all animals upon study completion. A single-cell suspension was prepared from spleen and depth inguinal draining lymph nodes sampled at necropsy in order to perform ELISPOT analysis.

Cell preparation. Bone marrow-derived DCs (BMDCs) were prepared from C57BL/6 mice as previously described (35) and used at day 6 of culture. MoDCs were prepared from buffy coats of normal blood donors. Briefly, PBMCs were isolated on a Ficoll density gradient (GE Healthcare) and resuspended into RPMI-1640 glutamax medium. Monocytes were then isolated by aggregation at cold temperature, and contaminating $\mathrm{T}$ cells were removed by rosette formation after overnight incubation at $4^{\circ} \mathrm{C}$ with an equal volume of sheep red blood cells. Another Ficoll density gradient was then performed to isolate enriched monocytes, which were resuspended and maintained in complete RPMI medium supplemented with 1,000 U/ml GM-CSF and $400 \mathrm{U} / \mathrm{ml} \mathrm{IL}-4$ (Miltenyi Biotech). Fresh complete medium was replaced after 4 days, and immature MoDCs were obtained after 7 days of culture.

TILs were purified from collected tumor tissues. Briefly, tumor tissues were cut into small pieces, and resuspended in HBSS medium containing $2 \mathrm{mg} / \mathrm{ml}$ of collagenase D (Roche) and $100 \mu \mathrm{g} / \mathrm{ml}$ of DNase 
(Sigma-Aldrich). Tumors were digested for 1 hour at room temperature with magnetic stirring. FCS was then added to stop the enzymatic action, and tumor tissues were transferred to ice. A mechanic dissociation of the tumor was then performed on a $70-\mu \mathrm{m}$ cell strainer with a syringe plunger. Next, Percoll gradient (Pharmacia Biotech) was used to obtain a single-cell suspension.

Peripheral blood and spleen mononuclear cell suspensions from mice were isolated using Ficoll-Paque gradient (GE Healthcare) before flow cytometry analysis or ELISPOT assay.

PBMC suspension from NHPs was isolated using 90\% Ficoll-Paque gradient with $10 \%$ PBS.

In vitro DC activation. Human immature MoDCs plated in 24-well plates were loaded with the vaccine, MPLA (Avanti Polar Lipids) as a positive control $(300 \mathrm{nM})$ or with buffer and were incubated overnight at $37^{\circ} \mathrm{C}$. After cell scraping and washes, cells were stained for the monitoring of HLA-DR, CD80, CD83, and CD86 expression by flow cytometry. An activation index was calculated as follows: the geometric mean fluorescence intensity for each marker was reported and transformed as a percentage of the expression monitored for the positive control after subtracted the value observed for buffer-incubated cells. An average of the values obtained for each activation marker was calculated and used as the activation index.

Murine BMDCs were loaded with vaccine, MPLA (300 nM) as a positive control or buffer, and incubated overnight. Cell activation was monitored by flow cytometry after staining with anti-CD11c, anti-IA-IE, anti-CD86, and anti-CD40. An activation index was also calculated based on the expression level of IA-IE, CD86, and CD40 on CD11 ${ }^{+}$cells.

In vitro T cell stimulation. PBMCs from NHPs were counted and $2 \times 10^{6}$ cells were plated per condition. Cells were incubated with a pool of overlapping peptides for each of the 4 antigens of ATP125, with PHA as a positive control or without any stimulant as a negative control, in the presence of brefeldin $\mathrm{A}$ and anti-CD107a for 5 hours. After washing, cells were stained for cell-surface antigens CD3, CD4, CD8, and fixable viability dye. Next, after fixation and permeabilization, cells were stained for intracellular TNF- $\alpha$, IL-2, and IFN- $\gamma$. In parallel, $2 \times 10^{6}$ cells were plated in the presence of overlapping peptides from the 4 antigens of ATP125, or with 2 concentrations of concanavalin A (20 $\mu \mathrm{g} / \mathrm{ml}$ and $4 \mu \mathrm{g} / \mathrm{ml})$ and incubated for 6 days. Cells were then stained for surface antigens CD3, CD4, CD8, and fixable viability dye. After fixation and permeabilization, cells were stained for Ki-67 expression.

TLR activation experiment. HEK-Blue hTLR 2 cells and HEK-Blue hTLR4 cells were incubated with 1 $\mu \mathrm{M}$ of vaccine construct. After 24 hours, supernatants were recovered and IL- 8 measured by ELISA (R\&D Systems). BMDCs were incubated with $1 \mu \mathrm{M}$ of construct. After 24 hours, supernatants were harvested and IL-6 measured by ELISA (Biolegend). THP1-XBlue-MD2-CD14 cells were stimulated with various concentrations of vaccine constructs. Upon TLR stimulation, these cells produce the secreted embryonic alkaline phosphatase (SEAP), which is detectable using QUANTI-Blue Solution (InvivoGen). After 18 hours, cell supernatants were recovered and incubated with QUANTI-Blue Solution at $37^{\circ} \mathrm{C}$ according to manufacturer's instructions. SEAP activity was assessed by reading the optical density (OD) at $620 \mathrm{~nm}$.

Antibodies and flow cytometry. For surface staining of murine blood cells, the following mAbs were used: CD4 FITC (RMA4-4), CD11b FITC (M1/70), CD19 FITC (6D5), CD8 APC-H7 (53-6.7), KLRG1 BV421 (2F1), and PD-1 PE-Cy7 (29F.1A12) (all from BD Biosciences except PD-1, which is from Biolegend). Dead cells were identified with LIVE/DEAD yellow fluorescent reactive dye (L34959) from Life Technologies and were excluded from analyses. Murine MHC-peptide PE-conjugated multimers were from Proimmune or from Immudex. Multimer gating strategy used a dump gate (CD4, CD11b, CD19), a CD8 gate, and excluded dead cells.

Intracellular staining of murine spleen cells was performed after stimulation with the indicated peptides and in the presence of CD107a BV711 mAb (1D4B, BD Biosciences) for 6 hours in the presence of Brefeldin A (GolgiPlug, BD Biosciences). Intracellular staining was done with mAbs to IFN- $\gamma$ APC (XMG1.2, BD Biosciences), TNF- $\alpha$ PE (MP6-XT22, BD Biosciences), IL-2 Alexa Fluor 488 (JES6-5H4, $\mathrm{BD}$ Biosciences), and corresponding isotype controls (BD Biosciences). Fixation and permeabilization was carried out using the BD Bioscience kit according to manufacturer's instructions.

BMDCs were stained with CD11c PE (HL3), IA-IE BV421 (M5/114.15.2), CD40 APC (3/23), CD86 PE-Cy7 (GL1), and Live Dead Yellow (ThermoFisher Scientific).

Human monocyte-derived DCs were stained with CD1a PE (H1149), HLA-DR PE-Cy5 (BB7.2), CD80 BV605 (L307.4), CD83 FITC (HB15e), and CD86 APC (2331) (all from BD Biosciences) and Live Dead Yellow.

For ATP125 internalization monitoring, cells were stained with anti-6His antibody (clone 6His, BioLegend) unconjugated or FITC-conjugated on the cell surface, following the fixation-permeabilization step (CytoFix-CytoPerm kit, BD Biosciences) according to the manufacturer's instructions. For the extracellu- 
lar-only condition, the cell surface was stained with the FITC-conjugated anti-6His, for the intracellular-only condition, the cell surface was stained with the unconjugated anti-6His, and the intracellular compartment was stained with the FITC-conjugated anti-6His. For the extra and intracellular condition, both the cell surface and the intracellular compartment were stained with the FITC-conjugated anti-6His.

For staining of NHP cells, the following antibodies were used: CD45 FITC (D058-1283), CD4 BV605 (L200), CD123 BV786 (7G3), CD14 BV650 (M5E2), CD20 V450 (L27), CD8 PerCP-Cy5.5 (RPA-T8), CD16 APC-H7 (3G8), CD3 PE-Cy7 (SP34-2), HLA-DR PE-Cy5 (G46-6), CD28 APC (CD28.2), CD95 BV421 (DX2), CD56 PE (MY31), CD123 BV786 (7G3), CD107a FITC (H4A3), IL-2 FITC (MQ1-17H12), Ki-67 Alexa Fluor 488 (B56), and TNF- $\alpha$ PerCP-Cy5.5 (MAb11) from BD Biosciences and PD1 APC-Cy7 (EH12.2H7), IFN- $\gamma$ APC (4S.B3), and CD11c APC (S-HCL-3), from Biolegend.

Fixation and permeabilization was performed using the CytoFix-CytoPerm kit (BD Biosciences) according to the manufacturer's instructions. Cells were analyzed using a Gallios flow cytometer (Beckman Coulter) or an LSR II flow cytometer (BD Biosciences) and results were processed with FlowJo (TreeStar Inc.) or Kaluza (Beckman Coulter) software.

ELISPOT assay. The ELISPOT assay for the detection of peptide-specific IFN- $\gamma$-secreting T cells was performed essentially as described previously (36). Briefly, mouse splenocytes were incubated overnight in coated ELISPOT plates (Diaclone) in the presence or absence of a $5-\mu \mathrm{M}$ peptide epitope. In the same manner, cynomolgus splenocytes were incubated overnight in precoated Elispot plates (MabTeck) in the presence or absence of a pool of 15 amino acid-long peptides ( $5 \mu \mathrm{M}$ each), with an 11 amino acid-long overlapping region for the 4 antigens because cynomolgus animals were outbred with unknown HLA types. The number of peptide-specific IFN- $\gamma$-producing cells was calculated by subtracting the number of IFN- $\gamma-$ secreting cells cultured without peptide from that obtained with cells cultured with peptide.

Quantification of cytokine production. The production of IL-8 cytokine was measured in supernatants from HEK-Blue hTLR2 cells and HEK-Blue hTLR4 cells, using commercial ELISA kits according to the manufacturer's recommendations (R\&D Systems). IL-6 in the supernatant of BMDC cultures was measured (commercial Biolegend ELISA kit), following the manufacturer's recommendations. A multiplex ProCartaPlex kit, including IFN- $\gamma$, IL-1b, IL-12p40, IL-2, IL-6, IL-8, MCP-1, TNF- $\alpha$, and IL-10 was used to measure cytokines from the cynomolgus monkey plasma samples.

Humoral immune response. Mice were bled at different time points after vaccination with Z13Mad5Anaxa. Antibody titers against Z13Mad5Anaxa were measured by ELISA. Plates were coated with $10 \mu \mathrm{g} / \mathrm{ml}$ of Z13Mad5Anaxa in PBS, overnight at $4^{\circ} \mathrm{C}$. After washing with PBS $0.05 \%$ Tween 20 and blocking with PBS $0.05 \%$ Tween 20 containing $1 \%$ bovine serum albumin, serial dilutions of sera were added to the plates and incubated at $37^{\circ} \mathrm{C}$ for 2 hours. After washing, the plates were incubated with peroxidase-conjugated goat antimouse IgG (catalog A90-131P, Bethyl). Cut-off was determined as the OD mean plus 4SD of unvaccinated mice. Antibody titer was determined as the last dilution factor for which the OD was the upper cut-off value.

Cynomolgus monkeys were bled at different time points after vaccination with ATP125. A similar ELISA assay to that described above for antibody quantification in mice was performed but ELISA plates were coated with $10 \mu \mathrm{g} / \mathrm{ml}$ of ATP125 in PBS, and HRP-conjugated goat anti-monkey IgG heavy and light chain antibodies were used for detection (catalog A140-102P).

ATP125-loaded human DC ligandome analysis. DCs from HLA-A2 ${ }^{+}$donors were prepared from leukapheresis products, cultured in complete RPMI medium with 2,500 U/ml GM-CSF (Miltenyi Biotech) and 1,000 U/ml IL-4 (Miltenyi Biotech). The immature DCs were loaded with $300 \mathrm{nM}$ ATP125, stimulated with MPLA $\left(1.4 \mu \mathrm{M}\right.$, Avanti Polar Lipids) or control buffer overnight at $37^{\circ} \mathrm{C}$ before being scraped and pelleted for further ligandome analysis. A small number of cells were kept for activation state analysis by flow cytometry. HLA class I and class II molecules were isolated using standard immunoaffinity purification as described previously $(37,38)$. In brief, the pan-HLA class I-specific mAb W6/32, the pan-HLA class II-specific mAb Tü-39, and the HLA-DR-specific mAb L243 (all produced in-house) were used to extract HLA ligands.

Peptide samples were separated by nanoflow high-performance liquid chromatography (RSLCnano, Thermo Fisher Scientific) using a 50- $\mu \mathrm{m} \times 25-\mathrm{cm}$ PepMap rapid separation liquid chromatography column (Thermo Fisher Scientific) and a gradient ranging from $2.4 \%-32.0 \%$ acetonitrile over 90 minutes. Eluting peptides were analyzed in an online-coupled LTQ Orbitrap Fusion Lumos mass spectrometer (Thermo Fisher Scientific) using a top-speed collision-induced dissociation fragmentation method for HLA class I or a higher-energy collisional dissociation fragmentation method for HLA class II. For data processing, the Proteome Discoverer 1.4 (Thermo Fisher Scientific) was used to integrate the search results of the 
SEQUEST HT search engine (University of Washington) (39) against the human proteome as catalogued in the Swiss-Prot database (20,279 reviewed protein sequences, September 27th 2013) combined with the construct ATP125 without enzymatic restriction. Precursor mass tolerance was set to $5 \mathrm{ppm}$ and fragment mass tolerance to $0.02 \mathrm{Da}$. Oxidized methionine was allowed as a dynamic modification. The FDR, estimated by the Percolator algorithm 2.04 (40), was limited to 5\% for HLA class I and 1\% for HLA class II. Peptide lengths were restricted to 8-12 amino acids for HLA class I and 8-25 amino acids for HLA class II. Protein inference was disabled, allowing for multiple protein annotations of peptides. To determine HLA class I binding peptides, the NetMHCpan-3.0 algorithm $(41,42)$ with a percentile binding rank below $2 \%$ and the SYFPEITHI (43) algorithm with a threshold of $50 \%$ of the maximum score were used.

Statistics. Statistical analyses were performed using Prism software (GraphPad) and considered statistically significant if $P<0.05$. Statistical tests used included unpaired 2-tailed $t$ test, 2-way ANOVA test, Kruskal-Wallis test, Mann-Whitney test, and log-rank test.

Study approval. Mouse studies have been reviewed and approved by the institutional and cantonal veterinary authorities in accordance with Swiss federal law on animal protection. Monkeys were enrolled in compliance with animal health regulation (council directive no. 2010/63/EU September, 22, 2010 on the protection of animals used for scientific purposes).

\section{Author contributions}

$\mathrm{EB}$ and $\mathrm{MD}$ designed the vaccine platform. $\mathrm{EB}$ and $\mathrm{MD}$ designed the syngeneic mouse experiments. JFM designed the in vitro experiments. AN and SS performed the ligandome analysis. EB, JFM, SC, WDBB, and ED performed the in vivo and in vitro experiments. EB, JFM, MLSR, PRW, and MD analyzed the data and wrote the manuscript.

\section{Acknowledgments}

This work was supported by Eurostar grant E!8989 HuVac and by the Commission for Technology and Innovation (grant CTI18520.1).

Address correspondence to: Madiha Derouazi, AMAL Therapeutics, c/o Fondation pour Recherches Médicales, Av. de la Roseraie 64, 1205 Geneva, Switzerland. Phone: 41.22.379.46.88; Email: madiha. derouazi@amaltherapeutics.com.

1. Guo C, Manjili MH, Subjeck JR, Sarkar D, Fisher PB, Wang XY. Therapeutic cancer vaccines: past, present, and future. $A d v$ Cancer Res. 2013;119:421-475.

2. Melief CJ, van Hall T, Arens R, Ossendorp F, van der Burg SH. Therapeutic cancer vaccines. J Clin Invest. 2015;125(9):3401-3412.

3. Dagogo-Jack I, et al. Circulating tumor DNA identifies EGFR coamplification as a mechanism of resistance to crizotinib in a patient with advanced MET-amplified lung adenocarcinoma. J Thorac Oncol. 2017;12(10):e155-e157.

4. Alizadeh AA, et al. Toward understanding and exploiting tumor heterogeneity. Nat Med. 2015;21(8):846-853.

5. Sampson $\mathrm{JH}$, et al. Immunologic escape after prolonged progression-free survival with epidermal growth factor receptor variant III peptide vaccination in patients with newly diagnosed glioblastoma. J Clin Oncol. 2010;28(31):4722-4729.

6. Grenier JM, Yeung ST, Khanna KM. Combination immunotherapy: taking cancer vaccines to the next level. Front Immunol. 2018;9:610.

7. Ribas A, Wolchok JD. Cancer immunotherapy using checkpoint blockade. Science. 2018;359(6382):1350-1355.

8. Rosalia RA, et al. Dendritic cells process synthetic long peptides better than whole protein, improving antigen presentation and T-cell activation. Eur J Immunol. 2013;43(10):2554-2565.

9. Zhang $\mathrm{H}$, et al. Comparing pooled peptides with intact protein for accessing cross-presentation pathways for protective $\mathrm{CD}^{+}$ and CD4+ T cells. J Biol Chem. 2009;284(14):9184-9191.

10. Derouazi M, et al. Novel cell-penetrating peptide-based vaccine induces robust $\mathrm{CD}^{+}$and $\mathrm{CD} 8^{+} \mathrm{T}$ cell-mediated antitumor immunity. Cancer Res. 2015;75(15):3020-3031.

11. Belnoue E, et al. Enhancing antitumor immune responses by optimized combinations of cell-penetrating peptide-based vaccines and adjuvants. Mol Ther. 2016;24(9):1675-1685.

12. Zom GG, Khan S, Filippov DV, Ossendorp F. TLR ligand-peptide conjugate vaccines: toward clinical application. Adv Immunol. 2012;114:177-201.

13. Khan S, et al. Distinct uptake mechanisms but similar intracellular processing of two different toll-like receptor ligand-peptide conjugates in dendritic cells. J Biol Chem. 2007;282(29):21145-21159.

14. Okamura Y, et al. The extra domain A of fibronectin activates Toll-like receptor 4. J Biol Chem. 2001;276(13):10229-10233.

15. Timmerman JM. Carrier protein conjugate vaccines: the "missing link" to improved antibody and CTL responses? Hum Vaccin. 2009;5(3):181-183.

16. McWilliams JA, et al. Age-dependent tolerance to an endogenous tumor-associated antigen. Vaccine. 2008;26(15):1863-1873.

17. Parker KH, Beury DW, Ostrand-Rosenberg S. Myeloid-derived suppressor cells: critical cells driving immune suppression in the 
tumor microenvironment. Adv Cancer Res. 2015;128:95-139.

18. Shalapour S, Karin M. Immunity, inflammation, and cancer: an eternal fight between good and evil. J Clin Invest. 2015;125(9):3347-3355.

19. Nasif WA, Lotfy M, El-Sayed IH, E1-Kenawy Ael-M, E1-Shahat M, El-Hak NG. Implications of CEA and p53 overexpression in the poor prognosis of colorectal cancer. Med Oncol. 2006;23(2):237-244.

20. Xu F, Liu F, Zhao H, An G, Feng G. Prognostic significance of Mucin antigen MUC1 in various human epithelial cancers: a meta-analysis. Medicine (Baltimore). 2015;94(50):e2286.

21. Nagorsen D, et al. T cell responses against tumor associated antigens and prognosis in colorectal cancer patients. $J$ Transl Med. 2005;3(1):3.

22. Kalliakmanis JG, et al. Survivin expression in colorectal carcinomas: correlations with clinicopathological parameters and survival. Dig Dis Sci. 2010;55(10):2958-2964.

23. Shedlock DJ, Shen H. Requirement for CD4 T cell help in generating functional CD8 T cell memory. Science. 2003;300(5617):337-339.

24. Borst J, Ahrends T, Bąbała N, Melief CJM, Kastenmüller W. CD4. Nat Rev Immunol. 2018;18(10):635-647.

25. Andersen BM, et al. Monomeric annexin A2 is an oxygen-regulated toll-like receptor 2 ligand and adjuvant. J Immunother Cancer. 2016;4:11.

26. Swisher JF, Burton N, Bacot SM, Vogel SN, Feldman GM. Annexin A2 tetramer activates human and murine macrophages through TLR4. Blood. 2010;115(3):549-558.

27. Asada H, et al. Significant antitumor effects obtained by autologous tumor cell vaccine engineered to secrete interleukin (IL)-12 and IL-18 by means of the EBV/lipoplex. Mol Ther. 2002;5(5 pt 1):609-616.

28. Uronen-Hansson H, Allen J, Osman M, Squires G, Klein N, Callard RE. Toll-like receptor 2 (TLR2) and TLR4 are present inside human dendritic cells, associated with microtubules and the Golgi apparatus but are not detectable on the cell surface: integrity of microtubules is required for interleukin-12 production in response to internalized bacteria. Immunology. 2004;111(2):173-178.

29. Zom GG, Filippov DV, van der Marel GA, Overkleeft HS, Melief CJ, Ossendorp F. Two in one: improving synthetic long peptide vaccines by combining antigen and adjuvant in one molecule. Oncoimmunology. 2014;3(7):e947892.

30. Larocca C, Schlom J. Viral vector-based therapeutic cancer vaccines. Cancer J. 2011;17(5):359-371.

31. Mangsbo SM, et al. Linking T cell epitopes to a common linear B cell epitope: a targeting and adjuvant strategy to improve T cell responses. Mol Immunol. 2018;93:115-124.

32. Fletcher EAK, et al. Formation of immune complexes with a tetanus-derived B cell epitope boosts human $\mathrm{T}$ cell responses to covalently linked peptides in an ex vivo blood loop system. J Immunol. 2018;201(1):87-97.

33. Herbst RS, et al. Predictive correlates of response to the anti-PD-L1 antibody MPDL3280A in cancer patients. Nature. 2014;515(7528):563-567.

34. Tumeh PC, et al. PD-1 blockade induces responses by inhibiting adaptive immune resistance. Nature. 2014;515(7528):568-571.

35. Santiago-Raber ML, et al. Type-I interferon receptor deficiency reduces lupus-like disease in NZB mice. J Exp Med. 2003;197(6):777-788.

36. Miyahira Y, et al. Quantification of antigen specific CD8 ${ }^{+}$T cells using an ELISPOT assay. J Immunol Methods. 1995;181(1):45-54

37. Kowalewski DJ, Stevanović S. Biochemical large-scale identification of MHC class I ligands. Methods Mol Biol. 2013;960:145-157.

38. Nelde A, et al. HLA ligandome analysis of primary chronic lymphocytic leukemia (CLL) cells under lenalidomide treatment confirms the suitability of lenalidomide for combination with T-cell-based immunotherapy. Oncoimmunology. 2018;7(4):e1316438.

39. Eng JK, McCormack AL, Yates JR. An approach to correlate tandem mass spectral data of peptides with amino acid sequences in a protein database. J Am Soc Mass Spectrom. 1994;5(11):976-989.

40. Käll L, Canterbury JD, Weston J, Noble WS, MacCoss MJ. Semi-supervised learning for peptide identification from shotgun proteomics datasets. Nat Methods. 2007;4(11):923-925.

41. Hoof I, et al. NetMHCpan, a method for MHC class I binding prediction beyond humans. Immunogenetics. 2009;61(1):1-13

42. Nielsen M, Andreatta M. NetMHCpan-3.0; improved prediction of binding to MHC class I molecules integrating information from multiple receptor and peptide length datasets. Genome Med. 2016;8(1):33.

43. Rammensee H, Bachmann J, Emmerich NP, Bachor OA, Stevanović S. SYFPEITHI: database for MHC ligands and peptide motifs. Immunogenetics. 1999;50(3-4):213-219. 